\title{
Experimental Measurement of ECH Deposition Broadening: Beyond Anoma- lous Transport
}

\author{
M. W. Brookman ${ }^{1, a}$, M. E. Austin ${ }^{1}$, K. W. Gentle ${ }^{1}$, C. C. Petty ${ }^{2}$, D. E. Ernst ${ }^{3}$, Y. Peysson ${ }^{4}$, J. Decker ${ }^{4}$, and K. \\ Barada $^{5}$ \\ ${ }^{1}$ Institute For Fusion Studies, University of Texas at Austin \\ ${ }^{2}$ General Atomics, San Diego, CA \\ ${ }^{3}$ PSFC - MIT \\ ${ }^{4}$ CEA - Cadarache \\ ${ }^{5}$ UCLA
}

\begin{abstract}
This work provides a first experimental measurement of broadened ECH deposition on the DIIID tokamak. As seen in theory[1] and simulation[2], refraction by edge density fluctuations shifts the path of RF waves, altering ECH and ECCD deposition. This paper reports on an initial experimental confirmation of broadened ECH deposition on DIII-D tokamak. $T_{e}$ measurements from a 48 channel 2nd Harmonic ECE Radiometer digitized at $500 \mathrm{kHz}$ are used with a set of broadened trial ECH deposition functions to calculate time-dependent, modulation-induced heat fluxes. The fitting of convective and diffusive transport to these fluxes allows different ECH deposition profiles to be compared. The best-fit ECH deposition produces reasonable transport coefficients which compare favorably with simulation. This method is applied to a set of L- and $\mathrm{H}$ - mode DIII-D discharges. Accounting for diffusive, convective, and coupled transport, the ECH deposition profile is found to be 2 to 3 times wider than predicted by TORAY-GA ray tracing.
\end{abstract}

\section{Introduction}

Injection of RF power at the electron cyclotron frequency is used for localized heating $(\mathrm{ECH})$ and current drive(ECCD) in tokamaks. EC deposition has been treated by $1 \mathrm{D}$ ray tracing and more complex full-wave calculations [3] and benchmarked against experimental deposition measurements[4]. Still, unresolved questions with significance for ITER remain. Anomalous transport has been credited for the discrepancy between ray tracing predictions of ECCD profiles and experimentally measured profiles, which are spatially broader [5] [6]. Work at TCV on ECCD [7] casts doubt on anomalous transport as a primary driver of broadening. Certain experimental signatures are expected from the fast electrons generated by ECCD. However, synthetic HXR on TCV is inconsistent with a significant diffusion of EC driven current.

Instead, turbulent edge density fluctuations, present in all tokamak plasmas, can defract RF waves traveling through them. Edge $\tilde{n}_{e}$ effects on RF propagation have been seen in theory[1] and computation [2]. These fluctuations are located in the edge $(\rho>9)$ and are driven by drift wave turbulence. They have a characteristic size on the order of $\sim 5 \mathrm{~mm}$ and amplitudes on the order of $\tilde{n}_{e} / n_{e} \sim$.1. Fluctuations interact with a range of frequencies, lower hybrid[8] with $\mathrm{f} \sim 5 \mathrm{GHz}$, as well as EC waves with $\mathrm{f} \sim 100 \mathrm{GHz}$. This edge interaction changes the in-

\footnotetext{
a e-mail: brookmanmw@fusion.gat.com
}

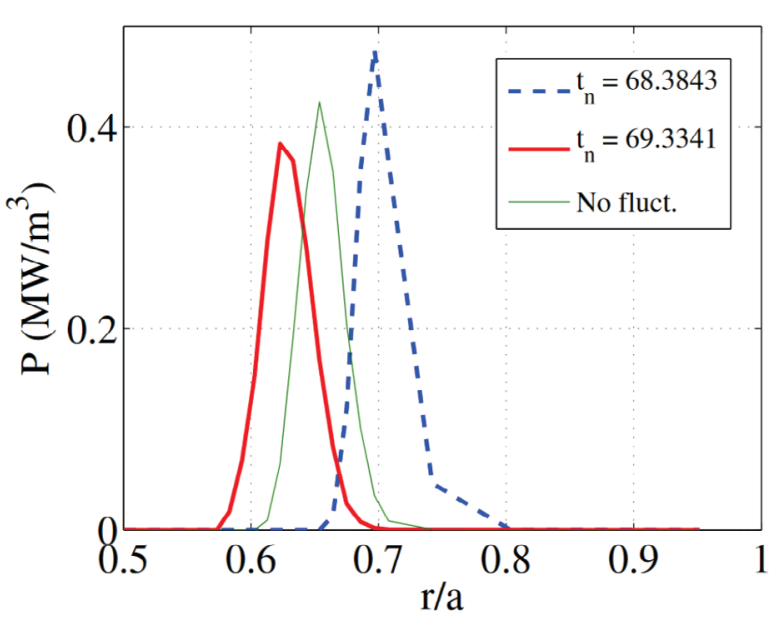

Figure 1. Results from Decker on TCV showing the ECH source function's range of variation over time due to edge fluctuations. Sufficiently rapid shifts compared to observation frequencies cause an effectively broader source function[7]/

tersection of the RF beam with the EC resonance on an edge turbulence timescale, $\tau<1 m s$. Fig. 1 shows an example of the defraction effect on the $P_{E C H}(r)$ deposition profile at different points in the same discharge. Observed by diagnostics, which have some finite response time, this appears as an effectively broadened profile. DIII-D experi- 


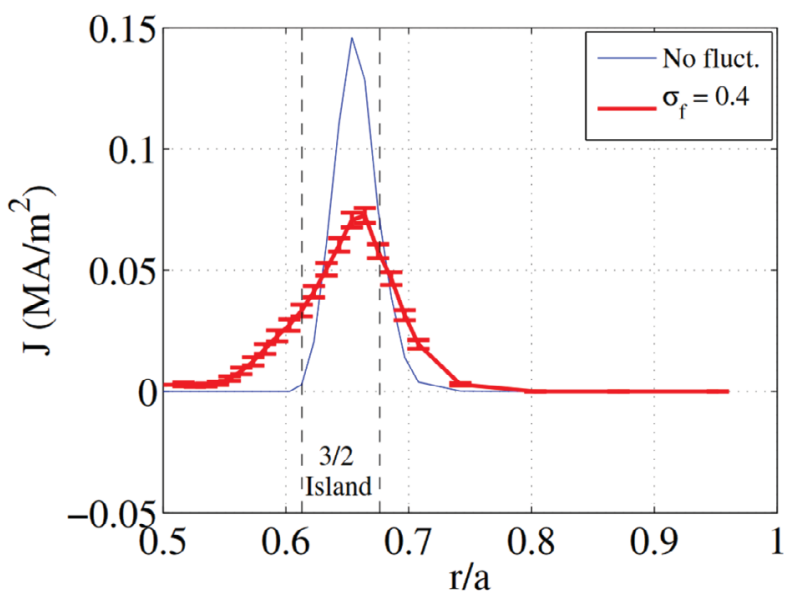

Figure 2. ECCD profiles predicted for ITER from the code $\mathrm{C} 3 \mathrm{PO} / \mathrm{LUKE}$ in the presence of modest edge fluctuation levels $\sigma_{f} \propto \tilde{n}_{e} / n_{e}$. Reprinted with kind permission of The European Physical Journal [7]

ments have also seen indications of broadening on the 110 $\mathrm{GHz}$ 2nd harmonic X-mode gyrotron system. Modulated ECH transport experiments by Gentle[10] found a hysteresis effect in critical gradient behavior, but this is minimal if $P_{E C H}(r)$ deposition is taken as $\sim \times 2$ wider than predicted by TORAY-GA.

ECCD and ECH experimental profile measurements are made through modulation, perturbing a discharge with a known input, and analyzing its effect on plasma parameters[4]. ECCD measurements can be made by Motional Stark Effect(MSE)[6]. MSE measures the change in pitch angle of magnetic field lines when ECCD is switched from co- to counter-current drive. This change in pitch can be analyzed to produce a driven current profile. The resistive timescales which govern current profiles limit ECCD measurement to slower modulations, no faster than $20 \mathrm{~Hz}$. This gives transport processes more time to obscure the instantaneous deposition profile, and because ECCD acts on a specific set of particles in phase space, this transport can be in both space and momentum [9] .

Measurement of ECH deposition through ECE is significantly simpler. Heat transport in tokamak plasmas can be treated in a radial 1D model[10]. Higher time and spatial resolution temperature measurements allow for faster modulation to be used, up to $1 \mathrm{kHz}$. Given that edge fluctuation broadening alters ECCD by means of altering ECH deposition location, ECE heat transport measurements offer a means of understanding this effect without the complexities of ECCD measurement.

It is vital to understand the impact of edge broadening on ITER. Altered ECCD deposition could hinder NTM suppression efforts, as the machine will need nearly its full power to suppress the predicted $3 / 2$ mode[11]. For a modest level of edge fluctuations, the LUKE/C3PO code (Fig. 2) predicts this effect will cause a significant amount of ECCD to fall outside the $3 / 2$ island. Current driven outside the island does not contribute to mode suppression.

\subsection{Overview}

In a series of discharges in the DIII-D tokamak an analysis of electron temperature data from ECE provides information about ECH deposition. For a best fit with linear coefficients, a significant level of deposition broadening is needed. This work explores a flux integral method of treating transport, performed in the Fourier basis of a modulated ECH power. A best fit $P_{E C H}(r)$ is found based on the relation between transport drive terms and heat flux. The flux is calculated (Sec. 2), and the method of transport fitting explored (Sec. 3.1). It is applied to set of DIII-D discharges with varying levels of edge turbulence (Sec.4.1). A 1.7 to 2.8 times broader deposition produces best fit and most reasonable transport coefficients.

\section{Resolving Deposition, Transport through Flux}

\subsection{D Heat Equation}

To understand deposition, it is necessary treat the timedependent heat flux. Perturbed by heating, transport moves ECH power promptly outside its initial deposition region. The $1 \mathrm{D}$ heat equation [13] provides the basis for calculating heat flux. It relates local stored energy of the plasma to heat and particle fluxes, sources, and sinks. Here $P_{E C H}$ is the ECH source function and $P_{O T H E R}$ includes contributions from e-i exchange, radiative, ohmic, and neutral beam power.

$$
\frac{3}{2} \frac{\partial}{\partial t}\left(n T_{e}\right)+\nabla \cdot\left(Q_{e}+\frac{5}{2} \Gamma T_{e}\right)=P_{E C H}+P_{O T H E R}
$$

The use of $50 \%$ duty cycle square wave modulated ECH power created a periodic change in plasma temperature. Linearizing the heat equation in this perturbation for fast modulation, $f_{\text {mod }}>20 \mathrm{~Hz}$ produces a simplified form. $P_{\text {OTHER }}$ is not directly modulated. Neutral beam power is constant and other indirectly modulated terms are small in amplitude according to ONETWO simulation of the discharges in this work. Based on Thomson scattering and reflectometry density measurements, for fast modulation $\tilde{T}_{e} / T_{e}>>\tilde{n}_{e} / n_{e} \approx 0$. Assuming the particle flux is negligible, a heat flux can be calculated from an experimental temperature and an ECH deposition profile.

$$
\tilde{Q}_{e}(r, t)=\frac{1}{r} \int_{r^{\prime}=0}^{r}\left(-\frac{3}{2} n_{e}\left(r^{\prime}\right) \frac{\partial \tilde{T}_{e}}{\partial t}\left(r^{\prime}, t\right)+\tilde{P}_{E C H}\left(r^{\prime}, t\right)\right) r^{\prime} d r
$$

\subsection{Fourier Analysis of ECE Temperature}

In the absence of transport (i.e $\tilde{Q}_{e}=0$ ), the local power deposition will be equal to the change in stored energy of the plasma, a time derivative of $T_{e}$. Rather than treat this derivative directly in experimental data, it is preferable to use Fourier analysis to produce a series of harmonic equations which reflect the time dependence. Expressing a Fourier transformed quantity with the 'hat' notation, 


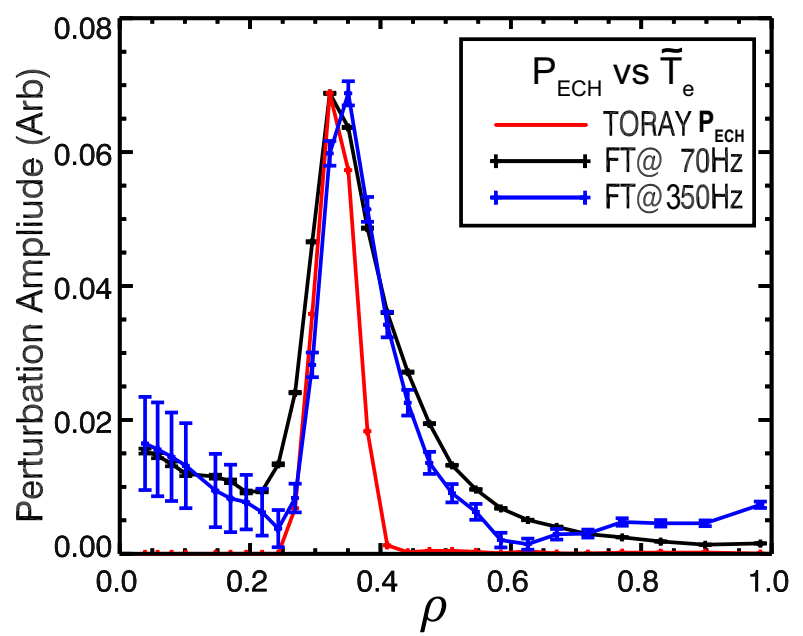

Figure 3. Normalized $\hat{T}_{e}$ perturbation profile produced with error bars by Fourier Analysis. 1st (black) and 5th (blue) harmonics. These are compared with the $P_{E C H}(r)$ source function predicted by the TORAY-GA ray tracing code [3].

$$
\frac{3}{2} n_{e} \frac{\partial \tilde{T}_{e}}{\partial t}(r, t) \approx \tilde{P}_{E C H}(r, t) \stackrel{D F T}{\longrightarrow} \frac{3 i \omega}{2} n_{e} \hat{T}_{e} \approx \hat{P}_{E C H}(r, f)
$$

$T_{e}$ data comes from a 48 channel 2 nd Harmonic Xmode ECE radiometer digitized at $500 \mathrm{kHz}$. A leastsquares fit Fourier series converts $48 T_{e}(\rho, t)$ signals over a $500 \mathrm{~ms}$ time period into a complex harmonic series in $n \times f_{\text {mod }}$. Odd harmonics dominate due to the squarewave input power. Faster timescale behavior is captured by higher frequencies, and thus the time dependence of the heat flux can be discerned. Error is propagated through the fit uncertainty of the Fourier harmonics, and reflectometryderived density, and the ECH source function terms.

Fig. 3 shows the 1 st and 5 th harmonic of the temperature perturbation as compared to a TORAY source function. The TORAY profile is substantially narrower, due to a combination of deposition broadening and transport. These will be distinguished through treatment of the timedependent flux in the Fourier basis of the perturbation[14].

Diffusive heat flux is driven by the temperature gradient, $\nabla \hat{T}_{e}$ using a high tension B-Spline, this term can be calculated from the perturbation profile. Fig. 4 shows ECE $T_{e}$ data from the 5th harmonic. A Monte Carlo integral can directly be taken on each part of the complex $\hat{T}_{e}$ and $\hat{P}_{E C H}$ expressed in a real and complex 'x+iy' formulation. An amplitude and phase ' $A e^{i \phi}$, integral has a significantly more complex form.

\subsection{ECH Source Functions}

The heat flux depends on experimental parameters, but also requires some assumed form of the input power. Past ECH transport work has often used a rectangular radial deposition function for analytic simplicity[17], but ray-tracing TORAY-GA, as well as C3PO/LUKE code from TCV[7], predict a smoother deposition. In lieu of LUKE/C3PO simulations for DIII-D, which are in progress,this work relies a series of trial blurred $P_{E C H}(r)$ source functions. As the edge effect occurs on a turbulence timescale 1-2 orders of magnitude faster than the ECH modulation. Thus the broadening can be expressed as an instantaneously broadened deposition characterized the parameter ' $b$ ', applied to the TORAY source function, $P_{E C H}(\rho, t)=P_{E C H}(\rho, b) \times P_{E C H}(t)$. Shown in Fig 4., the broadened deposition is created through a Gaussian blur in cylindrical coordinates. This blur can be characterized by a width $b$, such that

$$
P_{E C H}(\rho, b) \propto \rho^{-1}\left(\rho P_{T O R A Y}(\rho) * \exp \left(\frac{-\rho^{2}}{2 b^{2}}\right)\right)
$$

$\mathrm{ECH}$ input power does not form a perfect square wave. Gyrotrons have a finite switching time, and phases which vary by a few degrees, and thus the ECH does not posses a strictly $\sin (n 2 \pi f) / n$ character and the form of $P_{E C H}(t)$ must be considered. By performing the same Fourier series fit on the measured forward power from the gyrotrons, this flux integral fitting accounts for space and time variation of ECH in this flux calculation. This broadening and Fourier analysis are applied independently to each of the six gyrotrons to produce $\hat{P}_{E C H}(\rho, f)$ for calculating heat flux.

\subsection{Heat Fluxes Indicative of Broadening}

High-power ECH heating generates both an inward and outward traveling heat flux[17]. This constrains the phase and amplitude of $\hat{Q}_{e}$, and even before fitting transport the flux can help constrain the width of $P_{-E C H}$. Amplitude is small near the deposition center. Moving out from this region, a phase lag in $\hat{Q}_{e}$ reflects the time it takes for heat to propagate. Fig 6 shows a comparison of the amplitude and phase of the fundamental harmonic of $\hat{Q}(\rho, f)$ for various broadened input source functions- a TORAY source functions, a 2.3 times wider FWHM case and over-broadened 2.8 times wider case. In the next section, a 2.3 factor will be found to produce the best fit, and the corresponding flux shows a monotonic phase lag around the deposition. Higher frequencies reflect faster behavior, so higher harmonics should experience less transport and have a larger phase lag. Fig 7 shows a H-mode case with the correct broadening level. This H-mode has a significant ELMing character which perturbs the edge heat flux, but this perturbation does not reach into the core.

\section{Transport Fitting and Validation}

\subsection{Selecting Transport Coefficients}

Resolving ECH deposition requires treatment of transport. Convective and diffusive parts are needed to explain the heat flux induced by ECH[19]. $Q_{e}(\rho, t)=D n_{e} \nabla T+V n_{e} T$. Thus a modulated heat flux, $\tilde{Q}_{e}$, must be expressed as a linearizion in each parameter, including the transport coefficients. 

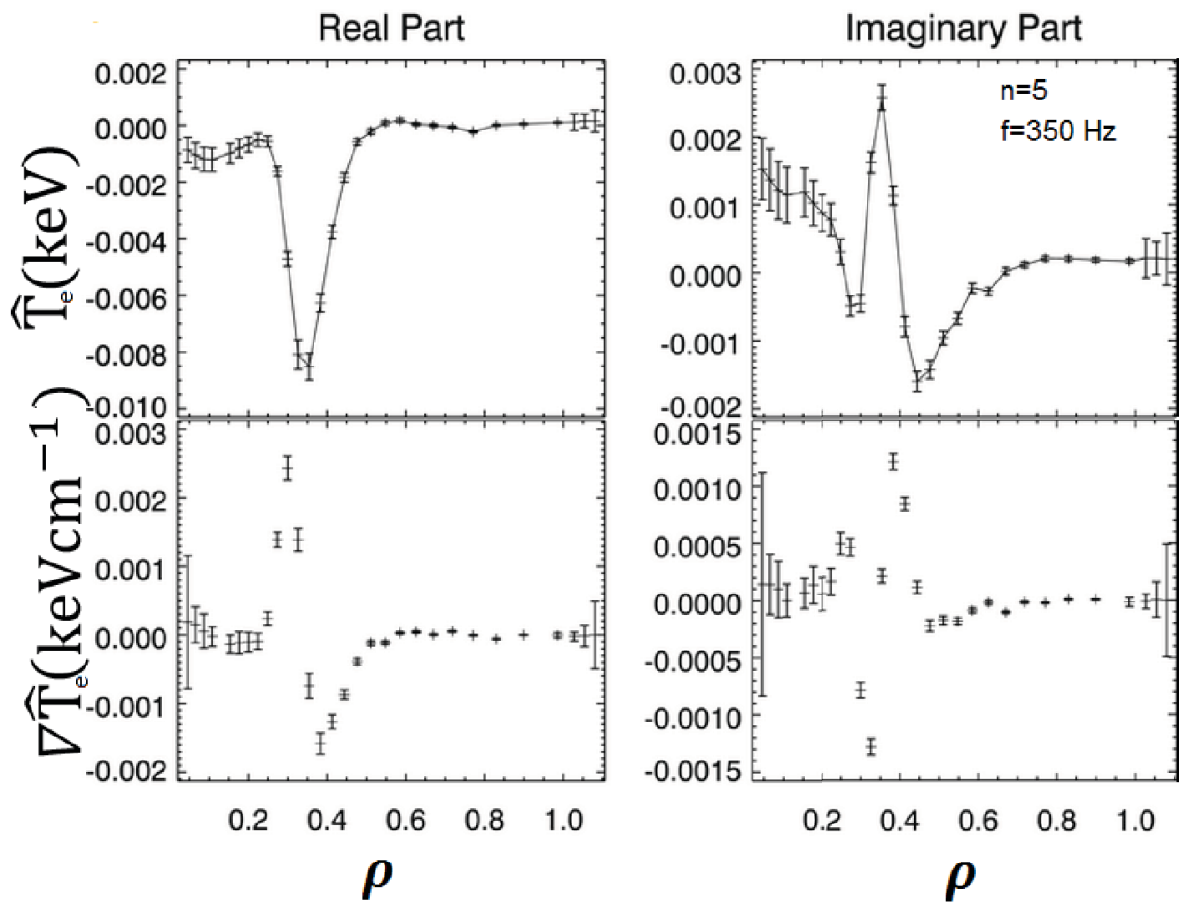

Figure 4. Real, imaginary parts of the perturbed temperature $\hat{T}_{e}$ as well as its gradient. The 5 th harmonic of the perturbation is shown, higher harmonics have larger uncertainties. Up to the 17 th harmonic, $1050 \mathrm{~Hz}$, can be resolved in this discharge.

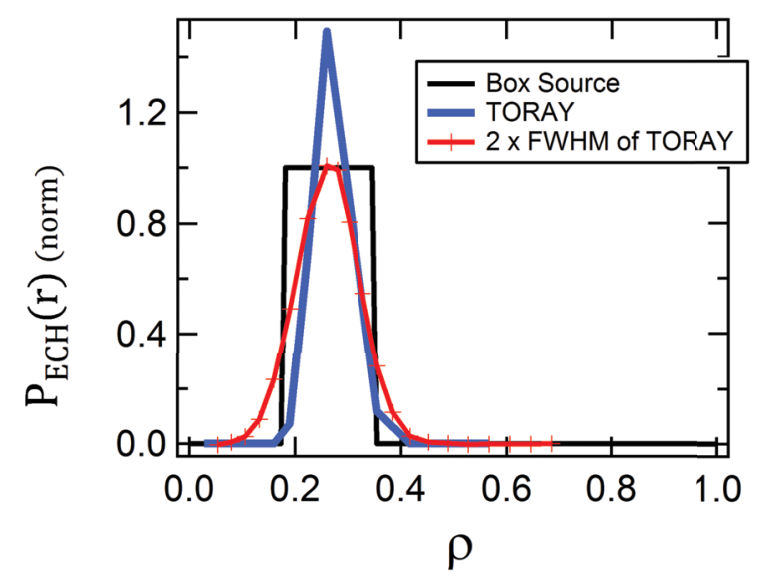

Figure 5. Comparison of possible source functions with the same total power. C3PO/LUKE calculations are not yet available for DIII-D, so broadened TORAY source functions are used.

$$
\tilde{Q}_{e}=n_{e}\left(D \nabla \tilde{T}_{e}+\tilde{D} \nabla T_{e}+V \tilde{T}_{e}+\tilde{V} T_{e}\right)+\tilde{n}_{e}(D \nabla T+V T)
$$

The time dependence of the modulated transport coefficients is indirect, entering through other plasma parameters which are periodically perturbed. Expressing this as an expansion for $\tilde{D}$ :

$$
\tilde{D}=\nabla \tilde{T}_{e} \frac{\partial D}{\partial \nabla T_{e}}+\tilde{T}_{e} \frac{\partial D}{\partial T_{e}}+\tilde{n}_{e} \frac{\partial D}{\partial n_{e}}+\nabla \tilde{n}_{e} \frac{\partial D}{\partial \nabla n_{e}}
$$

The terms which make up the modulated flux can be inserted and grouped into three distinct parts in the form $C 2 \nabla \tilde{T}_{e}+C 1 \tilde{T}_{e}+C 0 .[13]$

$$
\begin{array}{r}
\frac{\tilde{Q}_{e}}{n_{e}}=-\nabla \tilde{T}_{e}\left(D+\nabla T_{e} \frac{\partial D}{\partial \nabla T_{e}}\right)+\tilde{T}_{e}\left(V+T_{e} \frac{\partial V}{\partial T_{e}}-\nabla T_{e} \frac{\partial D}{\partial T_{e}}\right) \\
+\nabla \tilde{n}_{e}\left(T_{e} \frac{\partial V}{\nabla n_{e}}-\nabla T_{e} \frac{\partial D}{\nabla n_{e}}\right)+\tilde{n}_{e}\left(T_{e}\left(\frac{V}{n_{e}}+\frac{\partial V}{\partial n_{e}}\right)-\nabla T_{e}\left(\frac{D}{n_{e}}+\frac{\partial D}{\partial n_{e}}\right)\right)
\end{array}
$$

Consider $D_{M}=D+\nabla T_{e} \frac{\partial D}{\partial \nabla T_{e}}$ as a modulated diffusion driven by $\nabla \tilde{T}_{e}$. Likewise, $V_{M}=V+T_{e} \frac{\partial V}{\partial T_{e}}-\nabla T_{e} \frac{\partial D}{\partial T_{e}}$ is a modulated convection. The remaining term - driven by the modulated density and its gradient - will be grouped together as coupled transport, $\tilde{\xi}$. These simplifications produce a fitting equation of the form:

$$
\begin{aligned}
& \hat{Q}_{e}(r, f)=n_{e} D_{M} \nabla \hat{T}_{e}+V_{M} n_{e} \hat{T}_{e}+\hat{\xi} \\
& =\frac{1}{r} \int_{r^{\prime}=0}^{r} 3 i \pi f n_{e}\left(r^{\prime}\right) T_{e}\left(r^{\prime}, f\right)+P_{E C H}\left(r^{\prime}\right) \hat{P}_{E C H}(f) r^{\prime} d r
\end{aligned}
$$

\subsection{Treating Modulated Transport}

Constant diffusion and convection coefficients are insufficient to describe the full behavior of the time-dependent flux. Additional effects, collectively referred to as coupled transport, provide an additional contribution to the flux. 


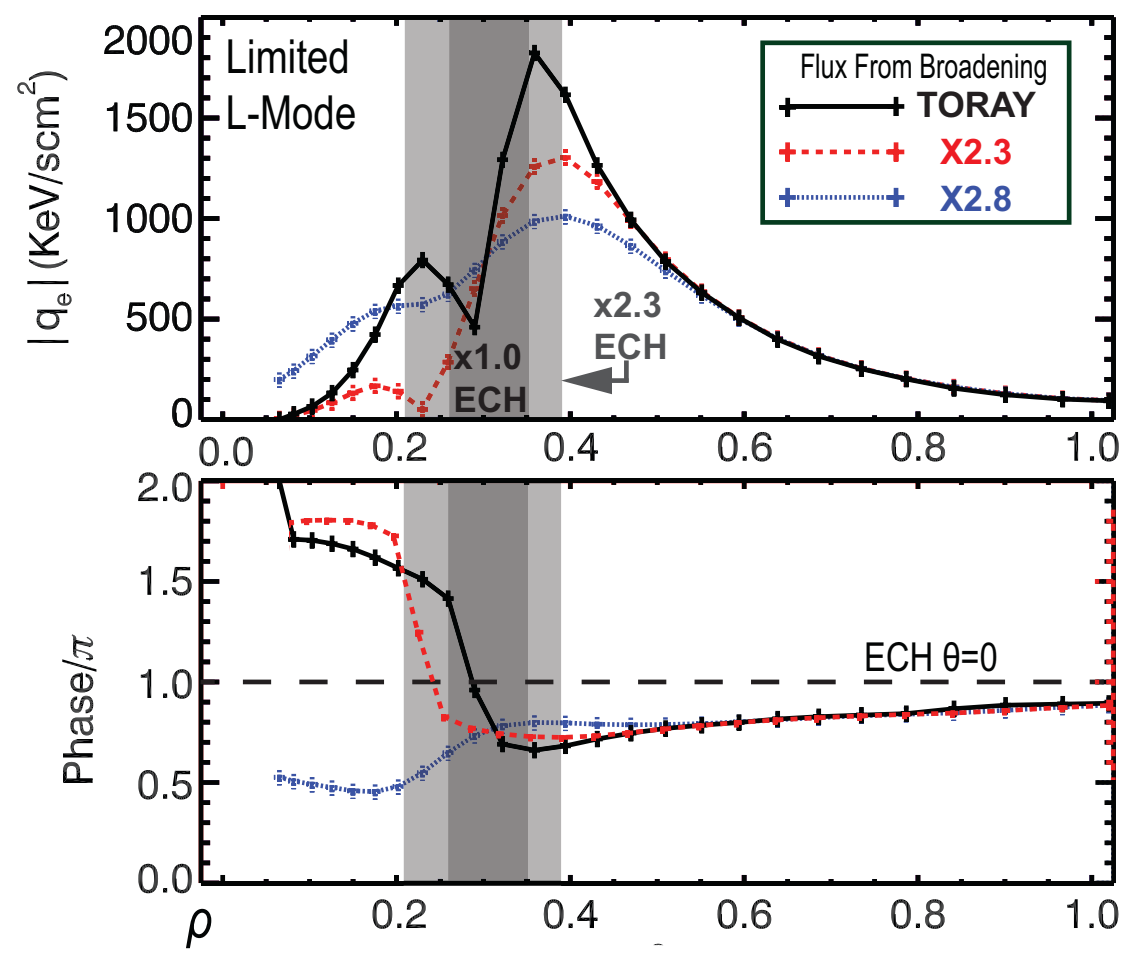

Figure 6. First harmonics of differently broadened heat fluxes from a limited L-Mode. A TORAY width source function overshoots in its phase change, as an artificially high flux from the narrow deposition region is needed to match the measured temperature perturbation. An overly broadened source, $\mathrm{x} 2.8$ wider, shows a very low phase in the core as an aphysical flux into the region is needed to correct the assumed $P_{E C H}$. A x2.2 wider source function, later found to be the best-fit, has a monotonic phase change across the deposition zone.

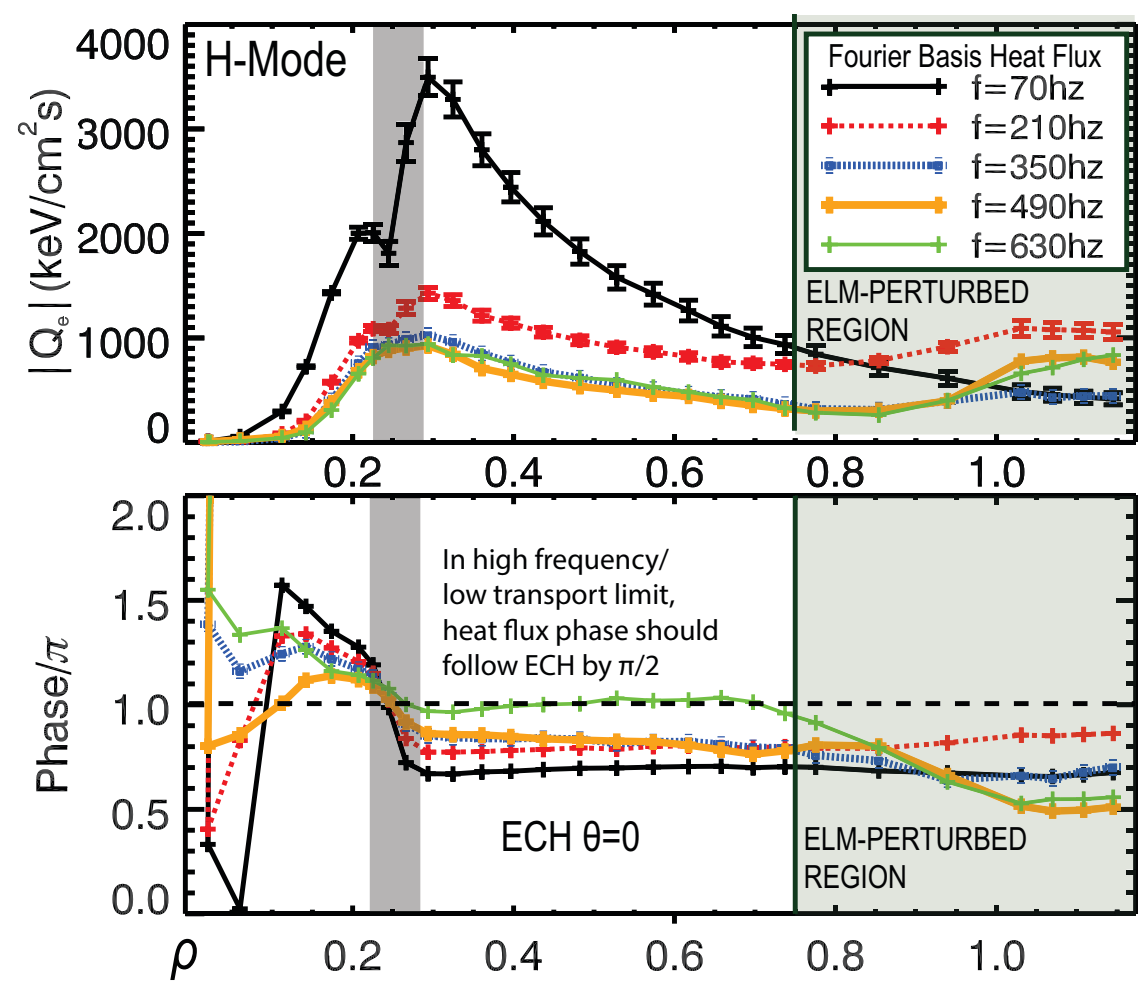

Figure 7. Heat flux calculated for a set of harmonics in an H-mode discharge with the best-fit source function. Inwards and outwards traveling heat pulses are clear from the phase at center of the deposition. The order of the phases inverts as lower frequency reflect slower changes. 
Since its drivers are not known a-priori, it must be treated as a fit parameter. $\hat{\xi}$ is a complex term with an unknown frequency dependence. Fitting to a variety of discharges has found the $\nabla T_{e}$ driven portion of $\hat{\xi}$ dominates. This makes $\hat{\xi}$ reminiscent of an anomalous diffusion[16].

$$
\hat{\xi} \approx-\nabla T_{e}\left(\nabla \tilde{n}_{e} \frac{\partial D}{\nabla n_{e}}+\tilde{n}_{e}\left(\frac{D}{n_{e}}+\frac{\partial D}{\partial n_{e}}\right)\right)
$$

Because fitting will be performed across harmonics, some form of time dependence must be applied, as $\nabla \tilde{n}_{e}$ and $\tilde{n}_{e}$ are not known. This is taken to be an exponential in time over a single modulation cycle -

$$
\tilde{\xi}(t) \propto \xi_{0} e^{-c\left(t-t_{0}\right)},\left\{t=\left(t_{0}, t_{0}+1 / f\right)\right\}
$$

This has a simple Fourier transform, which is the same up to a complex factor as that of the double-sided exponential decay. Thus we proceed with the form

$$
\hat{\xi}=\frac{C_{0} c e^{i \theta_{0}}}{c^{2}+4 \pi^{2} f^{2}} \nabla T_{e}
$$

The 3 fit parameters for this form are then an amplitude $C_{0}$, a phase $\theta_{0}$, and an exponential decay time c. There are three justifications for this form of diffusive-like coupled transport. Firstly, an exponential modulated diffusion appears frequently in the literature to fit heat pulse transport in experiments[16]. Time variation of $\hat{\xi}$ can be expressed in an exponential basis as well as a sine wave basis

$$
\hat{\xi}=\sum_{n} a_{n} \exp (-n t)
$$

Adding a full series of fit exponentials with variable phases would introduce a massive number of nonorthogonal fit parameters. Instead, this work fits a single decaying exponential form. A $\chi^{2}$ minimization will find the single frequency response which best accounts for time variation not driven by $D_{M}$ and $V_{M}$. Furthermore, this form seems to produce the best fit results. The imposed form of frequency fit produces a smaller $\chi^{2}$ result than a triangle or sawtooth wave time dependence, $\hat{\xi}(f) \propto 1 / f^{2}$ or $\hat{\xi}(f) \propto 1 / f$.

\subsection{Performing the Fit}

Equ. 5 has five unknown parameters - $D_{M}, V_{M}$, and the 3 parameters of $\hat{\xi}$ are fit to the sets of heat fluxes calculated for each of the trial $P_{E C H}$ deposition profiles. This avoids the challenge of conserving the total input power in a fit parameter form of $P_{E C H}$ which includes radial variation. Fits are performed using the uncertainties propagated in each parameter using orthogonal distance regression, ODR[18]. A traditional least-squares fit uses variation of an independent variable, here $\hat{Q}_{e} / n_{e}$, to minimize the quadrature difference between the calculated and fit values. The independent variables, $\nabla T_{e}, \nabla \hat{T}_{e}$, and $\hat{T}_{e}$ would need to be taken as exact. When fitting the heat equation both the independent and dependent variables have substantial known errors. $Q_{e}$ and its uncertainty are Monte Carlo integral quantities, not strictly local. Errors in $\nabla \hat{T}_{e}$ are larger than those in $\hat{T}_{e}$, but are of a similar magnitude. To treat these errors, ODR varies dependent variables as well, minimizing the uncertainty-weighted orthogonal separation between the data points.

The fit must also account for radial variation. Transport coefficients can change by an order of magnitude or more across the Tokamak. In the next section, results are presented from both locally constant fits made over a limited range of $\rho$ and from polynomial fit coefficients. Benchmarking shows that these two methods match within their uncertainties.

\section{Experimental Characterization of Broadening}

\section{1 $\chi^{2}$ Minimization \& Transport Coefficients}

This method has been applied to a set of discharges on the DIII-D Tokamak. The first of these was a wall-limited L-mode discharge where substantial broadening was expected. Fig. 8 shows a series of 5 th order polynomial fits of the radial variation of diffusion coefficients, and the resulting $\chi^{2}$ of the fit. The minimization of $\chi^{2}$ occurs for a source function with a FWHM x2.3 broader than predicted by TORAY-GA. The best fit source function produces a reasonable diffusion which is similar to the predicted diffusivity from the ONETWO transport code.

\subsection{Turbulence Comparison Through Matched H- and L- Mode Discharges}

Modulated ECH was injected into a series of DIII-D discharges in shape and density matched $\mathrm{H}$ - and L-mode discharges. Deposition was targeted at two locations, $\rho=$ 0.25 , and $\rho=0.6$. These targets have a significantly different path length through the plasma, but both generate inward and outward heat pulse propagation.

Two power levels, $3 \mathrm{MW}$ and 1.2 MW, were applied with $f_{\text {mod }}=70 \mathrm{~Hz}$. Density and deposition location are matched in the shapes, but improved H-mode confinement leads to a higher temperature. This broadens the ECH resonance, but this effect is already treated by the TORAYGA ray tracing code. Edge turbulence drops with the formation of the H-mode pedestal[20], allowing for a comparison of broadening in the same shape at two turbulence levels. Deposition and plasma profiles, as well as the Doppler backscattering channel measuring edge fluctuations for two matched core heating discharges are shown in Fig. 10. Transport analysis on these discharges has been performed, with a best-broadening polynomial $\mathrm{H}$-mode fit to $D_{M}$ and $V_{M}$ is shown in Fig. 9 for comparison with the earlier L-mode polynomial fit. $D_{M}$ compares very well with the $\chi_{e}$ diffusivity from ONETWO in the region where $V_{M}$ is small - where transport is dominated by diffusion and $\chi_{e} \approx D_{M}$. The ELM effects on the H-mode flux lead to unreasonable values of $D_{M}$ and $V_{M}$ in the edge.

To compare these discharges, constant coefficients were fit in the range $\rho=(.3, .6)$ for a series of broadening factors. The H-mode discharge shown in Table 1 and 

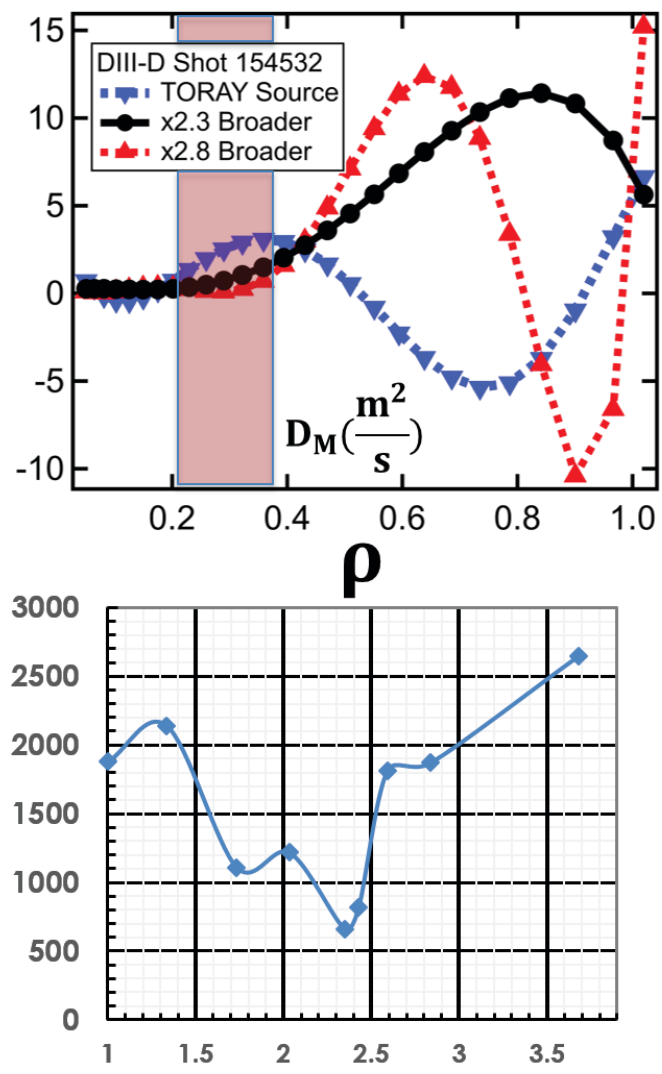

Figure 8. Physical values of $D_{M}$ for a Limited L-mode coincide with minimization of the $\chi^{2}$. A 5 th order polynomial can be used to represent radial variation in the Diffusion Coefficient (above). This can be compared to the ONETWO power balance diffusivity. The best fit occurs for a $\times 2.3$ broader FWHM for $P_{E C H}(r)$. This fits a consistently positive diffusion coefficient similar to the ONETWO value.

the L-mode in Table 2 show a clear minimization of $\chi^{2}$ and reasonable transport coefficient for broadened profiles, with L-mode requiring significantly more broadening than $\mathrm{H}$-mode. Also shown in these tables are the characteristic times, c, which define the best fit exponential decay. The two best-fit cases produce very similar characteristic timescale for coupled transport at the best-fit broadening factor.

\begin{tabular}{llll}
\hline Broad. Factor & $D_{M}\left(\mathrm{~cm}^{2} / s\right)$ & $c(1 / s)$ & $\chi^{2}$ \\
\hline$\times 1$ & $1.9 \pm .4$ & 423 & 2223 \\
$\times 2.0$ & $4.7 \pm .5$ & 386 & 520 \\
$\times 2.5$ & $4.6 \pm .4$ & 321 & 588 \\
$\times \mathbf{2 . 7 5}$ & $3.5 \pm .2$ & 293 & $\mathbf{3 4 6}$ \\
$\times 3.2$ & $3.6 \pm .2$ & -2844 & 555 \\
\hline
\end{tabular}

Table 1. The diverted L-mode fit from $\rho=(0.3,0.6)$ with a much larger broadening factor multiplied onto the very narrow

TORAY source. The FWHM of $P_{E C H}$ is still less than the limited L-mode above.

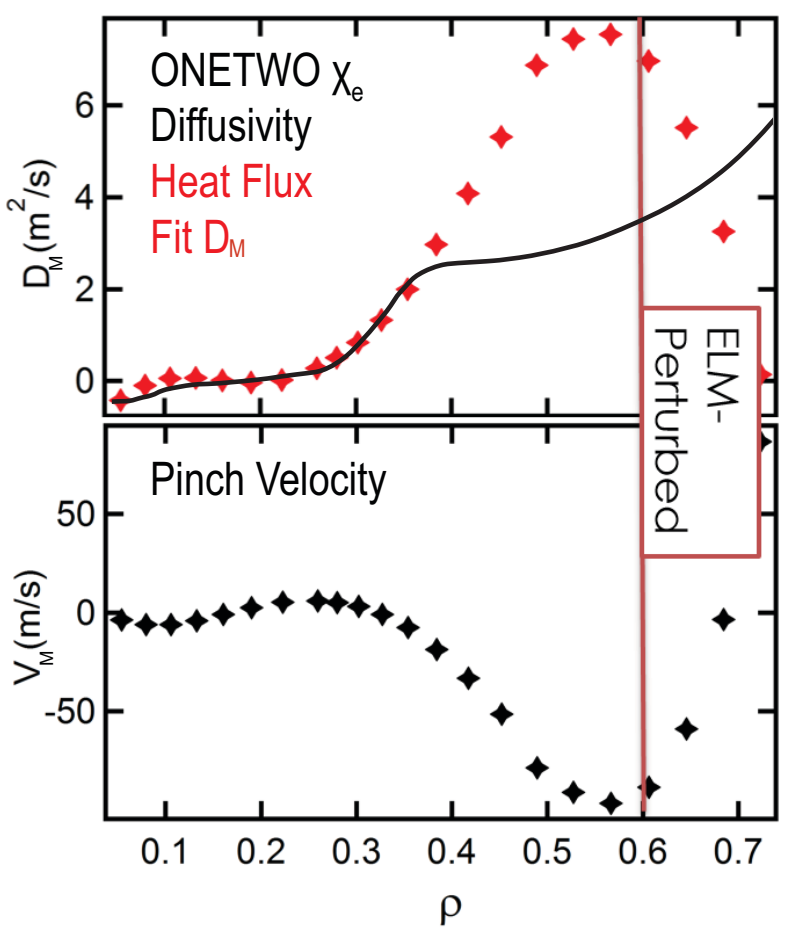

Figure 9. H-mode best fit transport coefficients are reasonable inside of $\rho=.6$. Outside this radius the effect of ELMs overwhelms the ECH heat flux, especially at high harmonics.

\begin{tabular}{llll}
\hline Broad. Factor & $D_{M}\left(\mathrm{~cm}^{2} / s\right)$ & $c(1 / \mathrm{s})$ & $\chi^{2}$ \\
\hline$\times 1$ & $3.2 \pm .1$ & 747 & 4067 \\
$\times 1.2$ & $1.2 \pm .1 .4$ & 2191 & 1223 \\
$\times 1.5$ & $1.4 \pm 1$ & 877 & 916 \\
$\times \mathbf{1 . 7}$ & $2.7 \pm 1.3$ & 306 & $\mathbf{2 2 3}$ \\
$\times 2.3$ & $5.4 \pm .8$ & 7025 & 393 \\
\hline
\end{tabular}

Table 2. Diverted H-mode discharge fits best from $\rho=(0.3,0.6)$ with a modest $\times 1.7$ broadening. Diffusion is comparable across cases to the ONETWO value for effective diffusivity

$$
\chi_{\text {eff }}=1.7-2.5
$$

\section{Conclusions and Future Work}

Calculation and fitting of modulated heat flux is a promising means of resolving ECH deposition. Best-fit transport coefficients are concurrent with significant ECH deposition broadening in both $\mathrm{H}$ - and L- mode predicted by TORAY-GA. For discharges which have a higher level edge turbulence, a substantially broader ECH deposition is found. These results are summarized in Table 3.

In future work, a causal connection between fluctuation levels and broadening factors will be explored, either through adjustment of edge fluctuations or a rigorous elimination of anomalous transport effects by adjusting targeting and plasma parameters. Other confinement modes, such as QH-mode and a recently achieved DIII-D negative triangularity L-mode, offer different edge turbulence conditions to be explored. LUKE/C3PO Simulations are underway for DIII-D, to produce computationally broadened profiles for use with the heat flux method. 

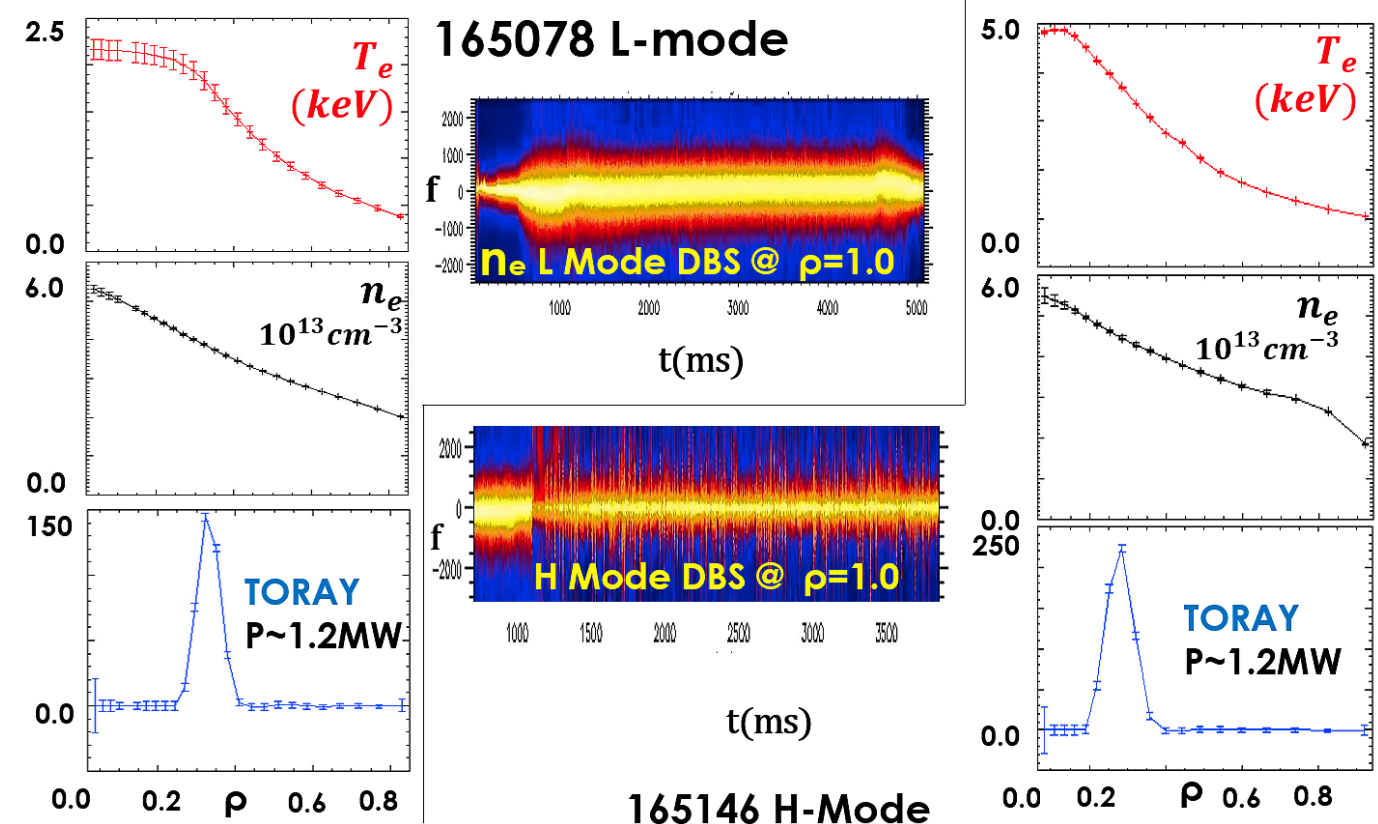

Figure 10. Profiles for the matched shape diverted H- and L- mode shots. The H-mode shows a clear reduction in edge density fluctuations as measured by DBS, which should lead to a drop in ECH broadening.

\begin{tabular}{llll}
\hline Discharge: & Limited L-mode & Diverted L-mode & Diverted H-mode \\
\hline TORAY FWHM & 0.072 & 0.055 & 0.067 \\
Best Fit FWHM & 0.17 & 0.15 & 0.11 \\
Factor & $\times 2.3$ & $\times 2.7$ & $\times 1.7$ \\
\hline
\end{tabular}

Table 3. FWHM of the best fitting broadened $P_{E C H}(r)$ from different shots. These discharge modes should have a steadily decreasing level of edge turbulence.

\section{References}

[1] Kyriakos H. \& Ram A.K. Phys. Plasmas 17, 022505 (2010)

[2] Peysson Y. et Al Plasma Phys. Control. Fusion 53, 124028 (2011)

[3] Prater R. et Al, Nucl. Fusion 48, 035006 (2008)

[4] Zerbini M. et Al, Plasma Phys. Control. Fusion 41, 931 (1999)

[5] Kirov K.K. et Al, Plasma Phys. Ctrl. Fusion 44, 2583 (2002)

[6] Petty C.C. et Al, Nucl. Fusion 42, 1366 (2002)

[7] Decker J. et Al, EPJ Web of Conf. 32, 01016 (2012)

[8] Peysson Y. \& Decker J. F.S.T 65, 22 (2014)

[9] P. Nikkola et Al, Nucl. Fusion 431343 (2003)

[10] Gentle K.W. et Al, Phys. Plasmas 13, 012311 (2006)

[11] LaHaye R.J. et Al, ECA 25A, 1357 (2001)

[12] Harvey R.W. et Al, Phys. Rev. Lett. 88, 205001 (2002)
[13] Gentle K.W. et Al, Phys. Fluids 31, 1105 (1988)

[14] Stockdale R.E. et Al, Bull. Am. Phys. Soc. 31, 1535 (1986)

[15] Peysson Y. et Al Plasma Phys. Control. Fusion 54, 045003 (2012)

[16] Fredrickson E. D., Phys. Rev. Lett. 65, 2869

[17] DeBoo J.C. et Al, Phys. Plasmas 19, 082518 (2012)

[18] Boggs P.T. et Al, Contemporary Mathematics 112, 186 (1990)

[19] Mantica P. et Al, Phys. Rev. Lett. 95, 185002 (2005)

[20] Rettig C. L. et Al, Nucl. Fusion 33643 (1993)

This material is based upon work supported by the U.S. Department of Energy, Office of Science, Office of Fusion Energy Sciences, using the DIII-D National Fusion Facility, a DOE Office of Science user facility, under Award DEFC02-04ER54698 and DE-FG03-97ER54415. DIII-D data shown in this paper can be obtained in digital format by following the links at https://fusion.gat.com/global/D3D $\_D M P$ 